\title{
The conceptual design of the electric scooter's steering-by-wire system
}

\author{
Fan Zhenzhen \\ Automotive Engineering institute, Jiangxi University of Technology, Nanchang 330098, China
}

Keywords: X-By-Wire; Electric scooter; Conceptual design

\begin{abstract}
Nowadays, big cities are faced with aging of the population and increasing physically disabled people caused by accidents. When those elderly people and disabled patients need others' support, assistance and care because of stiff knuckles, electric scooters show their advantages for being energy-saving and convenient. Therefore, electric scooters are developed as a mainstream. In the article, according to the controlling strategies of the system, a proper scheme controlled by the steering-by-wire system is confirmed. The controlling model for electric scooters driven by dynamo is developed and tested on the sample car.
\end{abstract}

\section{Introduction}

In the social activities, transport is an important part in people's daily life. Whether the transportation is convenient or energy-saving affects people's life quality to a great degree. Nowadays, big cities are faced with aging of the population and increasing physically disabled people caused by accidents. When those elderly people and disabled patients cannot behave at will because of rigid knuckles, what they need most is others' support, assistance and care. The current shortage of oil and urgency of environmental protection dominate the development of transportation, seeking for new low-noise and zero-emission vehicles which can use the energy synthetically. That is why electric scooters come into existence. At present, the electric vehicles on the market include electric cars, electric wheelchairs, electric bicycles, etc. Electric cars and wheelchairs are relatively expensive and inconvenient while electric bicycles and assistant vehicles are not comfortable and unsuitable for the elderly and the disabled. The electric scooter is a convenient, swift, energy-saving and environmentally friendly tool which combines human's own characteristics. It is widely accepted for its less pollution, low noise, simple structure and easy operation. As the scientific evidence shows, the electric scooter will be the leading role among the vehicle family in the near future and a quite promising manufacture industry.

The steering system of electric scooters is a special organ to change and maintain the driving direction. It helps drivers to change their directions based on their requirement and makes the vehicle function normally in the working conditions (straight driving, normal steering, quick steering, pivot steering) by cooperating with the traveling system. The traditional steering system of electric scooters is mechanical system. Drivers manipulate the steering wheel. Through the steering gear and a series of rod pieces, the steering movement is sent to the vehicle wheels. In recent years, owing to the development of electronic technology, the steering system adopts more and more electronic components. The electric scooter's Steering-By-Wire System (SBW) completely gets rid of the restraints of the traditional one because it cancels the mechanical joint between steering wheel and hand wheel, which can not only design the feature of the steering force transmission freely but also 
design the feature of the angle transmission, bringing forward great imagination. It is a significant innovation of the electric scooter's steering system.

\section{X-By-Wire technology}

X-By-Wire technology initially developed in the aerospace field. With the continuous improvement of the aircraft's flight speed and ceiling, traditional flight controlling system no longer fulfills the demand, which makes the X-By-Wire technology come into being. It generates so-called Fly-By-Wire system. Generally speaking, X-By-Wire technology is a new type that uses electric signals to control human's operation on the objects by electric wire (cable).

In recent years, the advantages of X-By-Wire technology meet the demand of highly-efficient, highly-precise and highly-secure control over vehicle's handling, thus making it inevitable to combine X-By-Wire technology with car's handling. The application of X-By-Wire technology to cars contains Steer-By-Wire, Brake-By-Wire, Throttle-By-Wire and Active Suspension (fig .1)

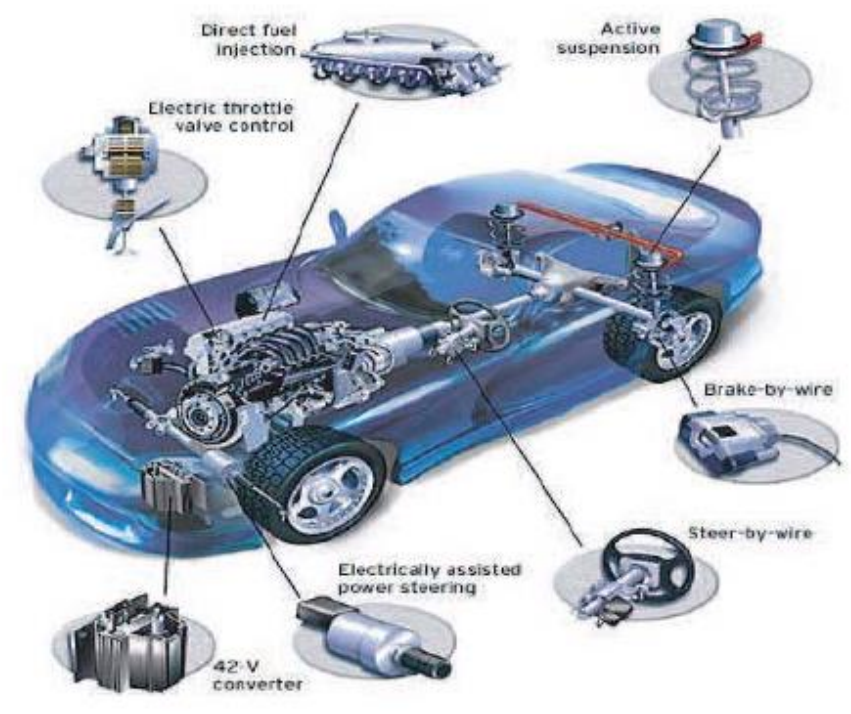

The Steering-By-Wire system consists of three principal parts (steering wheel assembly, steering performance assembly and ECU) and some assistant systems such as fail-safe system, power, etc. The steering wheel assembly includes the steering wheel, angle sensor, torque sensor, opposite rotary moment motor. The major function of the steering wheel assembly is to transform the driver's steering intention into digital signal which will be sent to the CU. Meanwhile, it receives the moment signal sent by ECU to generate opposite rotary moment of the steering wheel, so as to provide drivers with relevant information about road sense.

The vehicle-by-wire cancels the mechanical joint between the steering wheel and hand wheel by coordinating their movement through software. The mechanical restraint and interference are canceled so that it can move relatively independently, thus enabling to set the transmission ratio at random. For example, the program can set the transmission ratio according to the vehicle's driving condition based on the speed and the driver's likes. The steering-by-wire system is controlled by software. Thus, the steering system can be combined with other active safety devices such as ABS, vehicle dynamics control, anti-collision, track tracing, self-navigation, automatic driving, etc, so the vehicle can be controlled entirely and the overall stability can be improved. Besides, the vehicle's assistant steering function in ITS can be achieved. The steering-by-wire system is implemented through a universal actuator. If the vehicle adjusts its steering dynamics, an angle sensor must be 
utilized, which doesn't affect the quick adjustment of the wheels by steering wheel. On the other hand, a torque sensor will be used to adjust the car's steering and plays an important part in automatic driving.

The control by the electric scooter's steering system can be generally divided into two types. One is to focus on the configuration of the driving wheel, namely, in the form of the front passive wheel with rear driving wheel. Its shortcoming lies in the fact that it cannot be used in the place with narrow turning radius [28]. But in the real life, the users need to go outdoors as well as demand to live indoors. Therefore, the electric scooter suitable for narrow indoor conditions has been widely researched recently.

Steering-by-wire electric scooters adopt differential steering mainly composed of frame, steering driving mechanism, control mechanism and electronic circuit. The frame contains chassis and seat supporting mechanism. It eliminates the complex mechanical steering part of the traditional mechanical electric scooters. The chassis is designed as two elastic parts which can be jointed as a complete chassis when they slide and connect to each other parallelly. The dynamo drives the screw rod to make the stretch possible. The rear wheel of the frame is the driving wheel to control the scooter's movement and steering. The front wheel is universal wheel. Drivers can launch control signal through the mechanism. The signal is processed and sent to the driving mechanism through electric control circuit to control the direction and speed of the two dynamos so as to make the scooter turn in all directions.

\section{The conceptual design of X-By-Wire}

The steering-by-wire system replaces the mechanical rigid coupling between the traditional steering gear and wheels with electronic connection to use control signal to drive dynamo directly. It permits the design's encapsulation and modularization. The kinematics relationship of the steering gear and wheels is removed while it strengthens the control algorithm of the driver's input. As for the hardware, the steering-by-wire system can be divided into 3 parts: steering wheel system (steering wheel, steering angle sensors and mechanical transmission), CPU (dynamo driving module, signal gathering module and speed detection module) and steering driving system (driving dynamo, steering drive wheel).

The procedure goes as follows. CPU receives the control instruction form steering wheel and the signals gathered by sensors to identify its movement. CPU sends signal to control dynamo. The signal enables the circuit to drive dynamo to make the scooter to move in the given condition.

The omni direction move of electric scooter refers to the scooter's different movements and then returning to a new rectilinear motion by controlling the dynamo's corotation and reversal. At the beginning, the steering wheel rotates by some angle. A certain algorithm is used to distribute the speed of the two wheels to make the differential motion. The angle can be counted when the dynamo speed testing device detects the revolving speed of wheels on both sides respectively. It can be regarded as feedback quantity to ensure that the driving wheel angle accords with the input value. When the angle and input value fulfill the demand of accuracy requirement, the electric scooter will turn at the given angle. At last, redistribute an electric scooter with equivalent initial velocity to the new rectilinear motion.

Angle sensors are utilized to gather turning angles, which requires that they should be rather reliable and precise, so it is essential to select appropriate angle sensor chips for the steering-by-wire system. AS5040, produced by Austria Microsystems, is a sensor chip with non-contact and 
high-resolution coding to measure angles within $0^{\circ} \sim 360^{\circ}$. It has programmable increment output model for users with resolving ability of 10 bit. AS5040 permits the rotating speed to reach 10000 $\mathrm{r} / \mathrm{min}$, used to supply power of $3.3 \mathrm{~V}$ or $5 \mathrm{~V}$, whose working temperature range is $-40 \sim 125{ }^{\circ} \mathrm{C}$. The applications of AS5040 in industry are robots, motion control, brushless direct current motor reversing and electric tools. The applications in the vehicle engineering are steering wheel position sensor, gearbox encoder, headlight position control and power seat position sensor. Therefore, AS5040 has an advantage over other angle sensors for the steering wheel angle detection, high accuracy, and strong anti-jamming capability in the electric scooter's steering-by-wire system.

\section{Conclusion}

The application of X-By-Wire technology to the electric scooter's steering-by-wire system is studies in this article. Circuit-by-wire replaces the actuator's mechanical structure to drive dynamo directly by control signal, which gets rid of plentiful mechanical transmission mechanism. It can be proved that steering-by-wire technology can improve the steering executive efficiency of the electric scooter to ensure enough steering accuracy and make the drivers less exhausted. It has been improved compared with the traditional electric scooter's steering-by-wire system.

\section{Acknowledgements}

This work was financially supported by the key subject building project (vehicle engineering) of Jiangxi University of Technology.

\section{References}

[1] Lin C H. Novel adaptive recurrent Legendre neural network control for PMSM servo-drive electric scooter[J]. Journal of Dynamic Systems, Measurement, and Control, 2015, 137(1): 011010.

[2] Evans R, Bertrand J. Battery for electric scooter: U.S. Patent D709,824[P]. 2014-7-29.

[3] Siao B, Chang C. Electric scooter: U.S. Patent D720,660[P]. 2015-1-6.

[4] Lin C H. A novel hybrid recurrent wavelet neural network control of permanent magnet synchronous motor drive for electric scooter[J]. Turkish Journal of Electrical Engineering \& Computer Sciences, 2014, 22(4): 1056-1075.

[5] Lin C H. Dynamic control of V-belt continuously variable transmission-driven electric scooter using hybrid modified recurrent legendre neural network control system[J]. Nonlinear Dynamics, 2014: 1-22.

[6] Chen M C. Modular electric scooter frame structure: U.S. Patent Application 14/166,705[P]. 2014-1-28.

[7] Lin C H. Adaptive recurrent Chebyshev neural network control for PM synchronous motor servo - drive electric scooter with V - belt continuously variable transmission[J]. International Journal of Adaptive Control and Signal Processing, 2014.

[8] Maeyama Y, Omori H, Kimura N, et al. A novel type of super-rapid charging electric-motor driven scooter incorporating EDLC stack for home power appliances[C]//Power Electronics and Applications (EPE'14-ECCE Europe), 2014 16th European Conference on. IEEE, 2014: 1-6. 
[9] Lin C H. Novel adaptive modified recurrent Legendre neural network control for a PMSM servo-driven electric scooter with V-belt continuously variable transmission system dynamics[J]. Transactions of the Institute of Measurement and Control, 2014: 0142331214558105.

[10]Lin C H. Permanent magnet synchronous motor controlled V-belt continuously variable transmission driven electric scooter using hybrid modified recurrent Legendre NN control system[J]. INTERNATIONAL JOURNAL OF APPLIED ELECTROMAGNETICS AND MECHANICS, 2015, 47(1): 211-235. 